\title{
1. Introduction to the Handbook of Qualitative Research Methodologies in Workplace Contexts
}

\author{
Sarbari Bordia and Joanna Crossman
}

Welcome to the Edward Elgar Handbook of Qualitative Research Methodologies in Workplace Contexts. At the outset, we wish to thank the contributors and reviewers for their outstanding work in making this book a reality for all of us. We acknowledge that it was particularly difficult because of the challenges presented to us in late 2019 and all of 2020. Our Australian contributors had to endure the bush fire crisis, and following that, all our contributors had to deal with the pandemic. While these events caused some delay in the final stages of the book, we are delighted to present a suite of innovative chapters which, while adhering to traditional methodologies, address enquiries into the contemporary organisational context with novel approaches in qualitative research.

Qualitative methodologies and their respective tribes represent a broad church of assumptions within and amongst communities of practice. As editors, we are respectful of the diversity of qualitative research methodologies presented within the text and how our authors, as researchers, have identified themselves and their contributions upon a continuum of qualitative and quantitative perspectives. A handbook provides the opportunity to bring together these diverse perspectives in ways that other kinds of publications may not be able to do - but we are mindful that so vast is the intellectual terrain in qualitative research that we cannot capture its entirety.

The selected authors of chapters are unique individuals who draw upon the contexts of their own lives and experiences, living and working as they do across seven nations. Their contributions also reflect personal journeys in engaging with research work at different career points. Some are early or mid-career researchers and others are well-known and internationally respected as leaders, mentors and thinkers within their fields.

The Handbook is divided into three parts: Part I: Foundations; Part II: Innovations; and Part III: Applications. Quite obviously, the three parts of the Handbook are loosely applied since methodology, innovation and context seem much like distinguishing the seas of the world. We name them, take responsibility for them and ascribe jurisdiction, but in the end, all waters seem to flow into one another.

The chapters that make up Part I address some common and established methodologies from the perspective of their historical, philosophical and theoretical traditions. They also point to some of the issues that may be challenging while offering direction 
and guidance, as individual researchers exploring the same methodologies from sometimes different perspectives.

Part II presents contributions that are innovative, in that they offer insights into methodologies that are less known and utilised, mostly because they are still emerging as independent or distinct approaches. These approaches depart from, or can no longer be entirely reconciled with, well-established methodologies. These chapters, therefore, represent sites of tension, of questioning and exploration, inevitably leading to methodological innovations. The changing nature of workplaces, workers, contexts and researchers play important roles in the innovation of methodologies. We believe the beauty, and indeed, the effectiveness of qualitative research lie in the fact that such changes can be incorporated without compromising the core philosophical tenets of the research methodologies.

The contributions in Part III consider the reflexive use of the methodology. The place of the qualitative researcher is of particular importance in this section of the Handbook. Our role as qualitative researchers, our obligations, our identity, our tribes and the sometimes conflicting expectations that besiege us are important to the dynamic relationship amongst researchers, participants and research contexts. This part presents a set of chapters that explore the above-mentioned issues in the doing and teaching of qualitative research.

Overall, the chapters in the Handbook raise context as a significant issue for decision making about what constitutes an appropriate methodological choice. The nature of the context tests methodological alignment and raises questions about what processes are practicable or not. Context can invite the bricoleur in us, and compel us to make adaptations to established ways of conducting research when misalignments between context and methodology become apparent.

An understanding of the context demands that researchers engage in sense-making about the complex interactions between actors and environments in a consequent co-creation of knowledge that can destabilise assumptions, often unacknowledged, about the power and position of the researcher, largely by drawing upon reflexive techniques and insights. This reflexivity forces qualitative researchers to engage with the issues of culture, language, socio-economic status, education and almost every aspect of human experience that shapes the interpretation of data and creates narrative. Workplaces as contexts of human interaction offer the researcher innumerable, rich and varied possibilities for enquiry. This presents a very broad scope and one where a variety of disciplinary lenses may be relevant and so, in this way, the Handbook represents a multi-disciplinary work.

Diversity in qualitative research methodologies occurs because the space is a dynamic one where perspectives are continually contested as part of an intellectually healthy process supported in university policies and principles of academic freedom that provide a licence of sorts to ask risky questions and depart from conventional approaches towards new ways of thinking. Yet convention has its place. It provides necessary parameters in order to define and distinguish one methodology from another. It is necessary to understand the rules we make and break as members 
of particular research communities. In this respect, the Handbook represents the voices of both methodological gatekeepers and those who call them into question.

One of our aims in editing this book was to raise awareness of the ways in which qualitative research has responded to address the contemporary questions raised in workplaces. With the global dispersal of organisations, flexible work arrangements and changes to stakeholder relationships, the nature of workplace research has correspondingly shifted. Reflecting the dynamic landscape of workplaces and how they operate, contributors to the Handbook have, each in their own way, addressed contemporary issues reflexively and revealed insights into the implications for how qualitative researchers operate.

We anticipate too, in compiling this work during the Covid-19 pandemic, that workplaces will be changed in lasting ways, not least in the blurring of personal and professional realities as many employees necessarily work from home. The period will also likely lead to a greater emphasis upon online, remote data collection for qualitative researchers and on how unexpected, swift and extraordinary circumstances operating in a psyche of not knowing will promote creative approaches. This is why educators and researchers themselves need to foster ingenuity. It is our best hope for reaping long-term rewards in uncertain times when familiar, well-tested ways of conducting qualitative research become inoperable. The alternative is paralysis and a loss of relevance to the way we work.

We do hope you enjoy reading the Handbook and refer to it often, whether as a research candidate, an early career researcher or someone with an established research career, responsible for supervising and mentoring others. 